\title{
Marcadores neurobiológicos e psicométricos da eficácia da terapia cognitivo-comportamental no transtorno de estresse pós-traumático associado a sintomas dissociativos: relato de caso
}

\author{
Neurobiological and psychometric markers of the efficacy of cognitive behavioral therapy in \\ post-traumatic stress disorder with dissociative symptoms: a case report
}

\author{
Ana Cristina Lages ${ }^{1}$, Carlos Eduardo Nórte ${ }^{2}$, Ana Lúcia Pedrozo 3 , Raquel Menezes Gonçalves ${ }^{4}$, Carla \\ Marques-Portella ${ }^{5}$, Gabriela Guerra Leal Souza ${ }^{6}$, Ana Carolina Mendonça-de-Souza ${ }^{7}$, Paula Rui Ventura $^{8}$

\begin{abstract}
${ }^{1}$ Psicóloga, Universidade Federal do Rio de Janeiro (UFRJ), Rio de Janeiro, RJ. ${ }^{2}$ Mestrando do Instituto de Psiquiatria, UFRJ. ${ }^{3}$ Mestre em Psicologia, UFRJ. ${ }^{4}$ Doutoranda, Instituto de Psiquiatria, UFRJ. ${ }^{5}$ Doutora em Psiquiatria e Saúde Mental, UFRJ. Médica psiquiatra, Instituto de Psiquiatria, UFRJ. ${ }^{6}$ Doutora em Ciências Biológicas: Fisiologia, UFRJ. Professora adjunta, Instituto de Ciências Exatas e Biológicas, Universidade Federal de Ouro Preto (UFOP), Ouro Preto, MG. ${ }^{7}$ Doutora em Ciências Biológicas: Fisiologia, UFRJ. ${ }^{8}$ Doutora em Ciências Biológicas, UFRJ. Professora adjunta, Instituto de Psicologia e Instituto de Psiquiatria, UFRJ.
\end{abstract}

Suporte financeiro: Fundação do Amparo à Pesquisa do Estado do Rio de Janeiro (FAPERJ), Conselho Nacional de Desenvolvimento Científico e Tecnológico (CNPq), Financiadora de Estudos e Projetos (FINEP) e Coordenação de Aperfeiçoamento de Pessoal de Nível Superior (CAPES).

\section{Resumo}

Introdução: $\mathrm{O}$ transtorno de estresse pós-traumático (TEPT) tem sido associado à desregulação de diversos parâmetros psicofisiológicos e pode apresentar comorbidade com diversas psicopatologias, inclusive a dissociação, podendo dificultar o tratamento. O objetivo do presente trabalho foi investigar os efeitos da terapia cognitivo-comportamental em um paciente com TEPT e sintomas dissociativos com base em medidas psicofisiológicas e psicométricas em diferentes momentos do tratamento.

Descrição do caso: O paciente é um homem de 38 anos, com diagnósticos de TEPT e transtorno depressivo maior recorrente com sintomas dissociativos e resistência ao tratamento farmacológico. $\mathrm{O}$ tratamento psicoterápico teve duração de 4 meses, sendo composto por uma sessão semanal de terapia e três sessões semanais de coterapia. Os parâmetros fisiológicos (frequência cardíaca, tônus vagal, balanço simpático e cortisol) e psicométricos (afeto negativo e positivo, resiliência e sintomas de estresse pós-traumático, depressão, ansiedade e dissociação) foram medidos antes, durante (uma vez ao mês) e após o tratamento. Comentários: Os resultados mostraram diminuição do balanço simpático e aumento do tônus vagal, da frequência cardíaca e dos níveis de cortisol ao longo do tratamento. $\mathrm{O}$ tratamento também promoveu redução no afeto negativo e nos sintomas de estresse pós-traumático, depressão, ansiedade e dissociação, além de um aumento nos escores de resiliência, apoio social e afeto positivo. Esses resultados sugerem que a terapia cognitivocomportamental é um tratamento eficaz para o TEPT com sintomas dissociativos. Além disso, parece promover a normalização das alterações fisiológicas do sistema nervoso autônomo e neuroendócrino relacionadas ao TEPT.

Descritores: Transtornos de estresse pós-traumáticos, psicofisiologia, transtornos dissociativos, psicometria.

\begin{abstract}
Introduction: Post-traumatic stress disorder (PTSD) has been associated with dysregulation of many psychophysiological parameters and may present comorbidity with different psychopathologies, including dissociation, potentially affecting treatment outcome. The aim of this study was to evaluate the effects of cognitive behavioral therapy in a patient with PTSD and dissociative symptoms based on psychophysiological and psychometric measures at different time points throughout the course of treatment.

Case description: Our patient is a 38-year old man diagnosed with PTSD, major depression, and dissociative symptoms, resistant to pharmacotherapy. Psychological treatment lasted for 4 months and consisted of one weekly therapy session and three weekly coaching sessions. Physiological (heart rate, vagal tone, sympathovagal balance, and cortisol) and psychometric measures (negative and positive affect, resilience, and post-traumatic stress, depression, anxiety, and dissociative symptoms) were obtained before, during (once a month) and after treatment.

Comments: Results showed a decrease in sympathovagal balance and an increase in heart rate, vagal tone and cortisol levels during the course of treatment. Treatment also lead to a decrease in negative affect and in post-traumatic stress, depression, anxiety, and dissociation symptoms, as well as to an increase in resilience, social support and positive affect scores. These results suggest that cognitive behavioral therapy is an effective treatment for PTSD patients with dissociative symptoms. Moreover, the treatment seems to be able to normalize PTSD-related physiological parameters in the neuroendocrine and autonomic nervous systems. Keywords: Post-traumatic stress disorders, psychophysiology, dissociative disorders, psychometrics.
\end{abstract}

\section{Correspondência:}

Ana Cristina Lages Corrêa, Rua Bambina, 86/306, Botafogo, CEP 22290-140, Rio de Janeiro, RJ, Brasil. Tel.: (21) 2266.1465. E-mail: ana_lages85@yahoo.com.br Não foram declarados conflitos de interesse associados à publicação deste artigo. 


\section{Introdução}

Os achados de que indivíduos com transtorno de estresse pós-traumático (TEPT) apresentam parâmetros psicofisiológicos aumentados em resposta a estímulos relacionados ao trauma quando comparados com indivíduos sem TEPT levaram à inclusão, no Diagnostic and Statistical Manual of Mental Disorders, 3rd edition (DSM-III) ${ }^{1}$, da reatividade fisiológica como um critério sintomático formal para o diagnóstico de TEPT. Dessa forma, os pesquisadores começaram a questionar se pacientes diagnosticados com TEPT apresentariam níveis psicofisiológicos alterados mesmo na ausência de estímulos relacionados ao trauma. Pacientes com TEPT possuem maior ativação psicofisiológica em estado basal em relação aos indivíduos sem TEPT $^{2,3}$. A variabilidade da frequência cardíaca (VFC) tem sido proposta como um indicador de saúde física e mental e de regulação emocional, sendo que a baixa variabilidade indicaria deficiência de adaptação do sistema cardiovascular às demandas do organismo ${ }^{4}$. Recentemente, foi proposto que o aumento da frequência cardíaca (FC) em pacientes com TEPT pode ser mediado por redução da atividade parassimpática, e não pelo aumento da atividade simpática ${ }^{5}$.

Parâmetros neuroendócrinos associados à ativação do eixo hipotálamo-pituitária-adrenal também têm sido amplamente investigados nos estudos do estresse. A resposta do cortisol é considerada adaptativa ao estresse, e a desregulação desse eixo tem sido relacionada com uma série de patologias, entre elas o TEPT, em que os níveis de cortisol se encontram reduzidos ${ }^{6}$.

Sintomas dissociativos são relativamente comuns em pessoas que vivenciaram situações traumáticas ${ }^{7}$, apesar de não fazerem parte dos critérios diagnósticos para o TEPT de acordo com a quarta edição do DSM (DSM-IV) ${ }^{8}$. Indivíduos com TEPT e sintomas dissociativos formariam um subtipo dissociativo do transtorno, em oposição ao subtipo hiper-reativo 9 . As respostas biológicas ao estresse vivido numa situação traumática seriam distintas entre esses subtipos. Em estudo realizado com sobreviventes dos ataques de 11 de setembro de 2001, a dissociação foi relacionada a uma diminuída reatividade autonômica ao estresse e a menores níveis de cortisol ${ }^{10}$. Em um estudo de evocação de sintomas, somente os sujeitos que não dissociaram durante a revivescência tiveram aumento da $\mathrm{FC}^{11}$. Em outro estudo, menor FC basal foi observada em vítimas de acidentes com dissociação em comparação às vítimas sem dissociação ${ }^{12}$.

A terapia cognitivo-comportamental (TCC) é considerada eficaz no tratamento do $\mathrm{TEPT}^{13}$. Uma vez que há evidências na literatura de que a TCC pode promover mudanças neurobiológicas ${ }^{14}$, aspectos fisiológicos constituem instrumentos relevantes de avaliação de resposta ao tratamento e, por conseguinte, da eficácia da terapia ${ }^{15}$.

O objetivo deste trabalho foi investigar a eficácia da TCC no tratamento de um paciente com diagnóstico de TEPT e sintomas dissociativos que se mostrava resistente ao tratamento medicamentoso, com base na avaliação de medidas psicofisiológicas e psicométricas.

\section{Descrição do caso}

Descreve-se o caso de um paciente do sexo masculino, de 38 anos, que procurou atendimento no Instituto de Psiquiatria da Universidade Federal do Rio de Janeiro (UFRJ), em 2006, onde recebeu os diagnósticos de TEPT e transtorno depressivo maior recorrente, episódio atual moderado. Em 2005, uma mulher se jogou de uma passarela, na frente do ônibus que dirigia. Após o evento, passou a ter pesadelos e a lembrar-se com frequência da cena traumática. Após o trauma, também começou a apresentar sintomas dissociativos, como perder a noção do tempo e do espaço e sair de casa desorientado. $\mathrm{O}$ paciente evitava falar sobre o trauma, sair de casa e andar de ônibus, bem como situações, pessoas e lugares que lembrassem o acidente. Passou a apresentar irritabilidade, desânimo, tristeza, perda de prazer nas atividades habituais, insônia, fadiga, diminuição da concentração e impaciência. Também tinha crises agudas de ansiedade, com taquicardia e sudorese, e chegou a apresentar ideação suicida, além de alucinações visuais e auditivas.

O paciente foi medicado com antidepressivos em doses altas, paroxetina até $60 \mathrm{mg} /$ dia e venlafaxina até $375 \mathrm{mg} / \mathrm{dia}$, sem obter melhora expressiva. Foi, então, iniciado tratamento com tranilcipromina, aumentada progressivamente até se atingir a dose de $120 \mathrm{mg} /$ dia. Devido à ausência de resposta com o tratamento farmacológico, indicou-se associação deste com a TCC a fim de potencializar os resultados. Quando a TCC foi iniciada, em abril de 2008, o paciente utilizava as seguintes medicações: tranilcipromina $120 \mathrm{mg}$ por dia, clonazepam $3 \mathrm{mg}$ por dia, haloperidol $15 \mathrm{mg}$ por dia e trazodona $200 \mathrm{mg}$ por dia. O esquema medicamentoso se manteve estável ao longo de todo o tratamento psicoterápico.

$\mathrm{O}$ paciente assinou termo de consentimento aprovado pelo Comitê de Ética do Instituto de Psiquiatria da UFRJ ao início do estudo.

\section{Método}

\section{Protocolo de TCC}

Foi utilizado um protocolo adaptado de Foa \& Rothbaum ${ }^{16}$, com duração de 4 meses, composto por uma sessão semanal de terapia, conduzida por psicoterapeutas experientes, e três sessões semanais de coterapia, conduzidas por estudantes de psicologia. No total, foram 64 sessões de terapia e coterapia durante o tratamento. As técnicas utilizadas incluíram psicoeducação, reestruturação cognitiva, manejo de ansiedade e exposição imaginária e in vivo ${ }^{17}$.

\section{Avaliação psicométrica}

A avaliação psicométrica foi realizada em três etapas distintas: antes do início do tratamento (uma coleta), durante o tratamento (quatro coletas, sendo uma por mês) e após o tratamento (uma coleta). A avaliação consistiu no preenchimento de questionários relacionados a aspectos negativos, a saber: Inventário de Depressão de Beck (Beck Depression Inventory, BDI) ${ }^{18}$, Inventário de Ansie- 
dade de Beck (Beck Anxiety Inventory, BAI ${ }^{19}$, PTSD Checklist - Civilian Version (PCL-C) ${ }^{20}$, Escala de Experiências Dissociativas $(\mathrm{DES})^{21}$ e Escala de Traço de Afeto Negativo (PANAS-N) ${ }^{22}$. Também foram utilizados os seguintes questionários referentes a aspectos positivos: Escala de Traço de Afeto Positivo (PANAS$\mathrm{P}^{22}$, Escala de Resiliência (ER89) ${ }^{23}$ e Escala de Apoio Social do Medical Outcomes Study ${ }^{24}$. Todos os instrumentos foram utilizados em versões previamente validadas para o português.

\section{Avaliação psicofisiológica}

Os parâmetros psicofisiológicos foram avaliados em três etapas distintas: antes do início do tratamento (uma coleta), durante o tratamento (quatro coletas, sendo uma por mês) e após o tratamento (uma coleta). A avaliação consistiu em coleta de cortisol salivar e eletrocardiograma (ECG), com avaliação da FC, do índice parassimpático (VFC em altas frequências) e do índice simpático (balanço simpático). Para a análise dos níveis de cortisol, foram coletadas amostras de saliva utilizando o sistema Salivette ${ }^{\circledR}$. As amostras foram coletadas e armazenadas a $-20^{\circ} \mathrm{C}$ até o momento da análise. A dosagem foi realizada através do método de enzimaimunoensaio.

A aquisição e análise dos dados de ECG foram feitas utilizando-se os programas Acknowledge (Biopac Systems, Inc., Goleta, EUA) e Matlab 7.0 (MathWorks, Natick, EUA), respectivamente. Após a aquisição do sinal, foi utilizado um algoritmo para estimar as ondas $\mathrm{R} e$, posteriormente, foi realizada a inspeção visual do sinal para a correção de artefatos. As séries temporais foram reamostradas com interpolação a $2 \mathrm{~Hz}$, e foi gerado o período cardíaco. O período cardíaco (intervalo entre as ondas R do ECG) foi convertido para FC para facilitar a compreensão dos resultados. Foram realizadas análises espectrais utilizando a transformada rápida de Fourier para extrair a amplitude da potência espectral da VFC em altas frequências (VFC-AF: 0.15$0.4 \mathrm{~Hz}$ ) e em baixas frequências (VFC-BF: $0.05-0.15 \mathrm{~Hz}$ ). A VFC-AF medida em repouso tem sido associada à modulação parassimpática e, portanto, tem sido chamada de tônus vagal. A VFC-BF têm sido associada à modulação simpática e parassimpática. Dessa forma, o balanço simpático (índice gerado pela razão entre VFC-BF e VFC-AF) tem sido relacionado a uma modulação predominantemente simpática.

\section{Resultados}

Ao início das sessões de exposição in vivo, o paciente sentia ansiedade alta, que, com o enfrentamento, foi sendo lenta e gradativamente reduzida. Passou a sair mais, ir a lugares que evitava, sentindo-se mais tranquilo, e passou a conseguir conversar com as pessoas sobre o trauma, o que antes lhe causava grande desconforto. Antes da TCC, tinha as seguintes crenças: "sou um fracasso", "o mundo é perigoso" e "as pessoas não são confiáveis". Ao fim do tratamento, houve a substituição dessas crenças por outras mais realistas e adaptativas, como, por exemplo, "agora acho que fui vítima", evidenciando ausência de culpa pelo que aconteceu, e "quem é fraco não faz esse tratamento".
As escalas relacionadas a aspectos negativos mostraram reduções em sua pontuação ao longo do tratamento, resultado que foi mantido após seu término (Figura 1A). Já as escalas relacionadas a aspectos positivos mostraram aumento em suas pontuações (Figura 1B) durante e após o término do tratamento. Foi possível observar piora acentuada no mês 3 , com aumento da pontuacão nas escalas que avaliam aspectos negativos.

A

Aspectos negativos

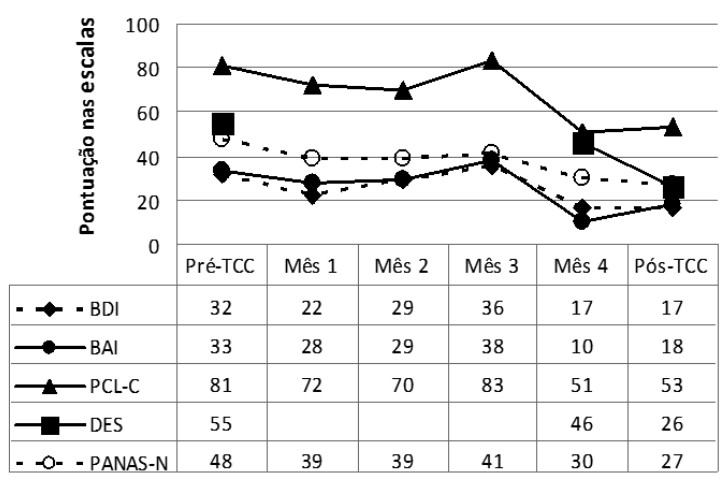

B

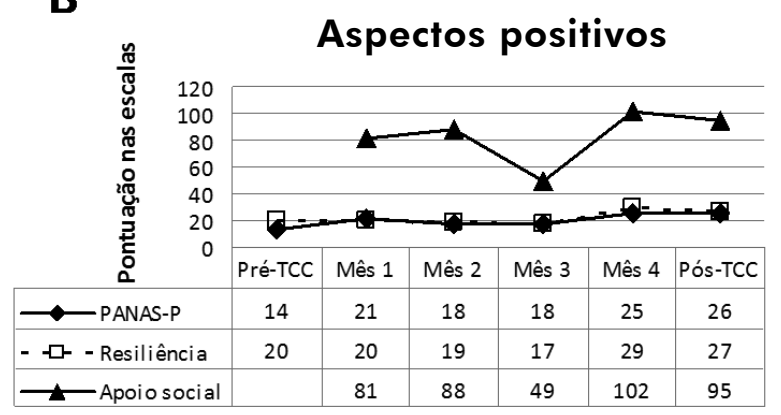

$\overline{\mathrm{TCC}}=$ terapia cognitivo-comportamental; BDI = Inventário de Depressão de Beck; BAI = Inventário de Ansiedade de Beck; PCL-C = PTSD Checklist - Civilian Version; DES = Escala de Experiências Dissociativas; PANAS-N = Escala de Traço de Afeto Negativo; PANAS-P = Escala de Traço de Afeto Positivo.

Figura 1 - Avaliação psicométrica a cada mês:

A) aspectos negativos; B) aspectos positivos

A comparação dos níveis basais de cortisol salivar antes $(6,36 \mathrm{nmol} / \mathrm{L})$ e após o término da terapia $(7,37 \mathrm{nmol} / \mathrm{L})$ evidenciou um pequeno aumento dos níveis hormonais. Além disso, foi observado aumento pronunciado nos níveis basais de cortisol durante o terceiro mês da terapia $(8,77 \mathrm{nmol} / \mathrm{L})$ (Figura 2A). Com relação à análise cardíaca, houve aumento progressivo da FC durante o tratamento, resultado que se manteve após seu término (FC antes da TCC: 76,6 bpm; FC após a TCC: 81,4 bpm) (Figura 2B). Foi observada também uma redução do tônus vagal no terceiro $\left(11,8 \mathrm{~ms}^{2}\right)$ e no quarto mês $\left(12,6 \mathrm{~ms}^{2}\right)$ em relação à coleta pré-TCC $\left(17,7 \mathrm{~ms}^{2}\right)$, porém esse quadro se reverteu para um aumento bastante acentuado na coleta pós-TCC $\left(32,0 \mathrm{~ms}^{2}\right.$ ) (Figura 2C). Com relação ao balanço simpático, houve aumento no primeiro mês $(7,1) \mathrm{em}$ relação à coleta pré-TCC $(3,2)$. Entretanto, nos meses seguintes, houve uma redução progressiva, até atingir os menores valores na coleta pós-TCC $(0,9)$ (Figura 2D). 
A

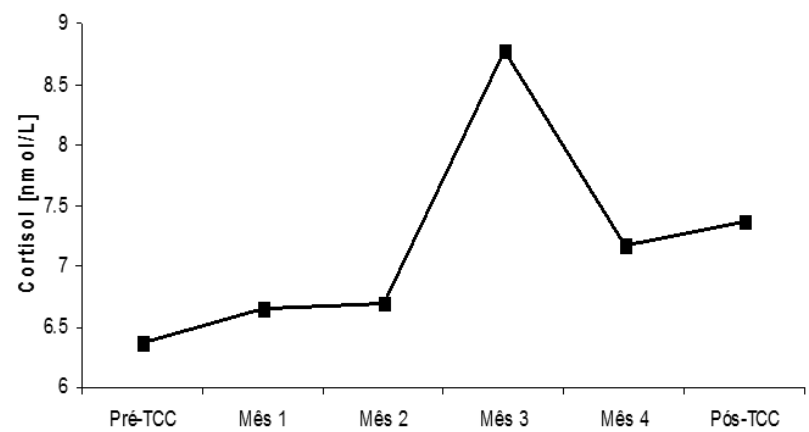

C

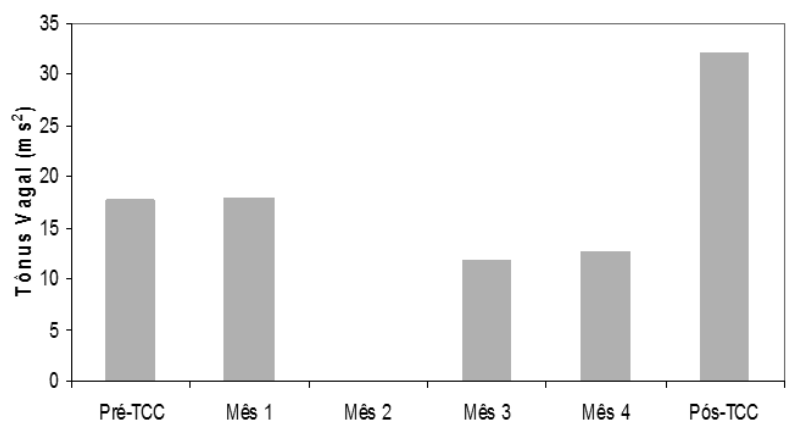

B

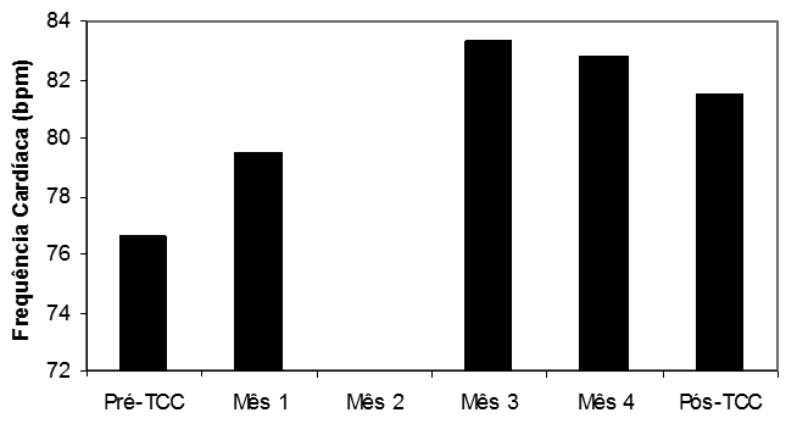

D

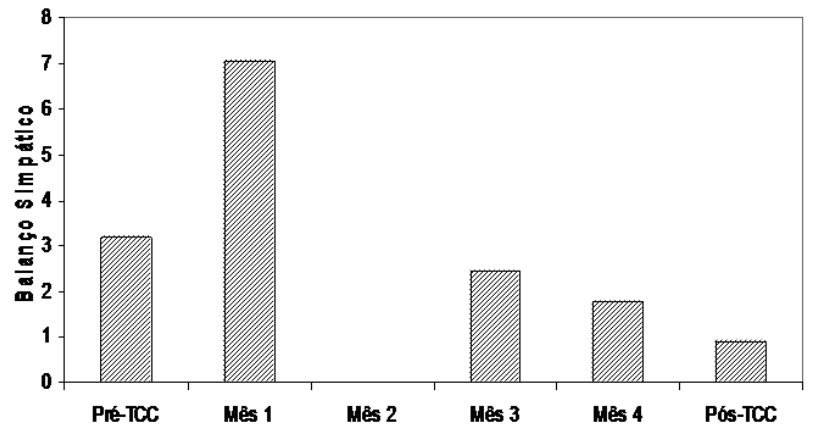

$\mathrm{TCC}=$ terapia cognitivo-comportamental.

Figura 2 - Parâmetros psicofisiológicos a cada mês: A) cortisol; B) frequência cardíaca; C) tônus vagal; D) balanço simpático

\section{Discussão}

O presente estudo fornece evidências sobre a eficácia da TCC para o tratamento de TEPT e sintomas dissociativos, visto que ocorreu um aumento nos escores das escalas que medem aspectos positivos e também das variáveis fisiológicas relacionadas a uma melhora da regulação emocional, associado a uma redução nos escores das escalas que medem aspectos negativos.

Houve redução dos sintomas dissociativos ao longo da psicoterapia, caracterizando ausência de dissociação. Esses dados sugerem uma relação entre o tratamento bem-sucedido do TEPT e a redução dos sintomas de dissociação, apesar de o protocolo não ter tido como foco o tratamento dos sintomas dissociativos. Tal resultado se contrapõe a estudos em que a TCC não foi eficaz para a redução dos sintomas dissociativos presentes em outros transtornos ${ }^{25,26}$.

Ao longo da terapia, notou-se uma piora significativa no terceiro mês da TCC. Essa piora caracterizou-se por uma queda acentuada na pontuação de escalas que avaliam aspectos positivos e um aumento nas pontuações das escalas que avaliam aspectos negativos. Concomitantemente, houve súbito aumento dos níveis de cortisol, atingindo seu valor máximo durante todo o tratamento, além de redução do tônus vagal. Durante o terceiro mês de terapia, o paciente se mostrou extremamente ansioso com as exposições. Uma vez que não ocorreram eventos negativos significativos em sua vida durante o terceiro mês de terapia que justificassem essa piora, consideramos que essas alterações seriam uma resposta ao próprio tratamento, mostrando uma forte associação entre a avaliação subjetiva e as respostas fisiológicas avaliadas no estudo. No quarto mês, os resultados psicométricos apontaram para um padrão de resposta mais saudável em relação aos meses anteriores, indicando uma possível habituação do paciente ao tratamento. Da mesma forma, no quarto mês, os níveis de cortisol caíram acentuadamente, voltando a aumentar ao fim do tratamento, embora de modo menos abrupto. Podemos inferir que essa melhora indicada pelo cortisol e pelas escalas reflete a queda da ansiedade perante as exposições, o que foi corroborado pela observação clínica dos terapeutas. É interessante notar que as alterações nos níveis de cortisol parecem acompanhar as alterações na avaliação psicométrica do paciente ao longo do tratamento. Apesar de sutil, o aumento nos níveis de cortisol no final do tratamento pode ser considerado um indicativo da eficácia da TCC, uma vez que vai a favor da literatura, que sugere níveis diminuídos de cortisol em pacientes com TEPT ${ }^{6}$ e em pacientes com dissociação após trauma ${ }^{10}$.

Apesar do aumento progressivo da FC ao longo do tratamento, houve redução do balanço simpático e aumento do tônus vagal. O sistema nervoso autônomo, particularmente o ramo parassimpático, tem um papel tanto na recuperação cardíaca quanto na promoção de resposta mais adaptativa ao estresse $e^{4,27}$. $\mathrm{O}$ aumento da FC pode ser indicador da redução dos sintomas dissociativos ao fim da TCC, uma vez que a literatura mostra que a FC é menor em pacientes traumatizados com dissocia- 
ção ${ }^{28}$. Tem sido proposto que indivíduos com maior tônus vagal possuem maior habilidade de se autotranquilizar em situações ameaçadoras, apresentam mais comportamento de engajamento social saudável ${ }^{4,27}$ e menor probabilidade de desenvolver transtornos psicopatológicos ${ }^{29}$. A literatura sugere, ainda, que o aumento do tônus vagal e possivelmente a redução do balanço simpático seriam marcadores biológicos de flexibilidade fisiológica e, consequentemente, bons índices de saúde física e mental. No presente estudo, o tratamento com a TCC parece ter contribuído diretamente para a promoção dessas alterações.

O acompanhamento clínico através da administração de escalas e da observação clínica foi incrementado pelo registro das variações fisiológicas na evolução do quadro do paciente. Por serem medidas objetivas, tais parâmetros podem ser indicadores precisos do quadro clínico, sendo menos vulneráveis a vieses ${ }^{30}$. A estabilização do tratamento farmacológico ao longo da TCC foi fundamental para a comparação dos parâmetros fisiológicos ao longo de todo o estudo. A associação das medidas fisiológicas aos indicadores psicométricos tradicionais, portanto, parece ser uma estratégia útil para o estudo da eficácia de tratamentos terapêuticos.

\section{Conclusão}

A associação da TCC ao tratamento farmacológico potencializou a remissão dos sintomas de TEPT e dos sintomas dissociativos do paciente, que não havia apresentado melhora sob tratamento unicamente farmacológico realizado durante 18 meses (paciente considerado resistente à medicação). Apesar dos evidentes ganhos terapêuticos, talvez a resposta ao tratamento não tenha sido ainda mais positiva devido ao foco exclusivo no quadro de estresse pós-traumático e à duração limitada do tratamento, de 16 semanas. Acreditamos que uma proposta de TCC que trate outros aspectos além do TEPT e que não tenha uma duração predeterminada poderia maximizar as respostas do paciente. Contudo, a TCC se mostrou eficaz e promoveu alterações psicofisiológicas importantes, as quais se refletiram em mudanças cognitivas e comportamentais, confirmando a presença de aspectos emocionais, cognitivos, comportamentais e fisiológicos no processamento do medo em pacientes com transtornos de ansiedade.

Esta pesquisa possui as limitações inerentes a um estudo de caso. A fim de melhor compreender o impacto da TCC sobre a fisiologia do TEPT, é imprescindível aumentar a amostra de pacientes, para que seja feito um estudo controlado randomizado.

\section{Referências}

1. American Psychiatric Association. Diagnostic and statistical manual of mental disorders, 3th ed (DSM-III). Washington: American Psychiatric Association; 1980.

2. Buckley TC, Kaloupek DG. A meta-analytic examination of basal cardiovascular activity in posttraumatic stress disorder. Biol Psychol. 2001;63:585-94.
3. Pole N. The psychophysiology of posttraumatic stress disorder: a meta-analysis Psychol Bull. 2007;133(5):725-46.

4. Porges SW. The polyvagal perspective. Biol Psychol. 2007;74:116-43.

5. Sack M, Hopper JW, Lamprecht F. Low respiratory sinus arrhythmia and prolonged psychophysiological arousal in posttraumatic stress disorder: heart rate dynamics and individual differences in arousal regulation. Biol Psychiatry. 2004;55(3):284-90.

6. Yehuda R, Morris A, Labinsky E, Zemelman S, Schmeidler J. Ten-year followup study of cortisol levels in aging holocaust survivors with and without PTSD. J Trauma Stress. 2007;20(5):757-61.

7. Carlier IV, Lamberts RD, Fouwels AJ, Gersons BP. PTSD in relation to dissociation in traumatized police officers. Am J Psychiatry. 1996;153(10):1325-8.

8. American Psychiatric Association. Diagnostic and statistical manual of mental disorders, 4th ed (DSM-IV). Washington: American Psychiatric Association; 1994.

9. Bremner D. Acute and chronic responses to psychological trauma: where do we go from here? Am J Psychiatry. 1999;156:349-51.

10. Simeon D, Yehuda R, Knutelska M, Schmeidler J. Dissociation versus posttraumatic stress: cortisol and physiological correlates in adults highly exposed to the World Trade Center attack on 9/11. Psychiatry Res. 2008;161(3):325-9.

11. Lanius R, Williamson P, Boksman K, Densmore M, Gupta M, Neufeld R, et al. Brain activation during script-driven imagery induced dissociative responses in PTSD: a functional magnetic resonance imaging investigation. Biol Psychiatry. 2002;52:305-11.

12. Bryant R, Harvey A, Guthrie R, Moulds M. A prospective study of psychophysiological arousal, acute stress disorder, and posttraumatic stress disorder. J Abnorm Psychol. 2000;109(2):341-4.

13. Mendes D, Feijó M, Ventura P, Passarela C, Mari J. A systematic review on the effectiveness of cognitive behavioral therapy for posttraumatic stress disorder. Int J Psychiatry Med. 2008;38(3):241-59.

14. Porto P, Oliveira L, Mari J, Volchan E, Figueira I, Ventura P. Does cognitive behavioral therapy change the brain? A systematic review of neuroimaging in anxiety disorders. J Neuropsychiatry Clin Neurosci. 2009;21(2):114-25.

15. Marmar C. Mental health impact of Afghanistan and Iraq deployment: meeting the challenge of a new generation of veterans. Depress Anxiety. 2009;26:493-7.

16. Foa E, Rothbaum B. Treating de trauma of rape: cognitive behavioral therapy for PTSD. New York: The Guilford Press; 1998.

17. Martins AL. Tratamento cognitivo-comportamental do transtorno de estresse póstraumático em pacientes resistentes à medicação [dissertação]. Rio de Janeiro: Universidade Federal do Rio de Janeiro; 2009.

18. Cunha JA. Beck Depression Inventory (BDI): manual da versão em português das escalas Beck. São Paulo: Casa do Psicólogo; 2001.

19. Beck A, Epstein N, Brown G, Steer RA. An inventory for measuring clinical anxiety: psychometric properties. J Consult Clin Psychol. 1988;56:893-7.

20. Berger W, Mendlowicz M, Souza W, Figueira I. Equivalência semântica da versão em português da Post-Traumatic Stress Disorder Checklist - Civilian Version (PCL-C) para rastreamento do transtorno de estresse pós-traumático. Rev Psiquiatr Rio Gd Sul. 2004;26(2):167-75.

21. Fiszman A, Cabizuca M, Lanfredi C, Figueira I. A adaptação transcultural para o português do instrumento Dissociative Experiences Scale para rastrear e quantificar os fenômenos dissociativos. Rev Bras Pisquiatr. 2004;26(3):164-73.

22. Watson D, Clark LA, Tellegen A. Development and validation of brief measures of positive and negative affect: the PANAS scales. J Pers Soc Psychol. 1998;54:1063-70.

23. Block J, Kremen AM. IQ and ego-resiliency: conceptual and empirical connections and separateness. J Pers Soc Psychol. 1996;70(2):349-61.

24. Griep R, Chor D, Faerstein E, Werneck G, Lopes C. Validade de constructo de escala de apoio social do Medical Outcomes Study adaptada para o português no Estudo Pró-Saúde. Cad Saude Publica. 2005;21(3):703-14.

25. Michelson L, June J, Vives A, Testa S, Marchione N. The role of trauma and dissociation in cognitive-behavioral psychotherapy outcome and maintenance for panic disorder with agoraphobia. Behav Res Ther. 1998;36:1011-50.

26. Rufer M, Held D, Cremer J, Fricke S, Moritz S, Peter H, et al. Dissociation as a predictor of cognitive behavior therapy outcome in patients with obsessivecompulsive disorder. Psychother Psychosom. 2006;75:40-6.

27. Porges SW. Cardiac vagal tone: a psychological index of stress. Neurosci Biobehav Rev. 1995;19(2):225-33.

28. Bryant R, Harvey A, Guthrie R, Moulds M. A prospective study of psychophysiological arousal, acute stress disorder, and posttraumatic stress disorder. J Abnorm Psychol. 2000;109(2):341-4.

29. Friedman BH, Thayer JF. Anxiety and autonomic flexibility: a cardiovascular approach. Biol Psychology. 1998;49:303-23.

30. Marmar C. Mental health impact of Afghanistan and Iraq deployment: meeting the challenge of a new generation of veterans. Depress Anxiety. 2009;26:493-7. 